NOTICE: this is the author's version of a work that was accepted for publication in Thin Solid Films. Changes resulting from the publishing process, such as peer review, editing, corrections, structural formatting, and other quality control mechanisms may not be reflected in this document. Changes may have been made to this work since it was submitted for publication. A definitive version was subsequently published in Thin Solid Films, Vol. 558 (2014).

DOI: 10.1016/j.tsf.2014.02.086 


\title{
A combinatorial investigation of sputtered Ta-Al-C thin films
}

\author{
Mark D. Tucker ${ }^{\text {b,a }}$, Marcela M. M. Bilek ${ }^{\mathrm{a}}$, David R. McKenzie ${ }^{\mathrm{a}}$ \\ ${ }^{a}$ School of Physics, The University of Sydney, NSW 2006, Australia \\ ${ }^{b}$ Thin Film Physics, Linköping University, 58183 Linköping, Sweden
}

\begin{abstract}
We describe a combinatorial experiment investigating the Ta-Al-C material system, conducted with the aim of determining why the tantalum-containing $\mathrm{M}_{n+1} \mathrm{AX}_{n}$ phases have so far proved to be not amenable to thin-film synthesis. Samples were deposited onto (0001) $\mathrm{Al}_{2} \mathrm{O}_{3}$ wafers at $8500^{\circ} \mathrm{C}$ and characterized by X-ray diffraction wafer maps, scanning electron microscopy, and surface optical scattering. Elemental Ta, the binary phases $\mathrm{TaC}, \mathrm{Ta}_{2} \mathrm{C}$, and $\mathrm{TaAl}_{3}$, and the ternary phases $\mathrm{Ta}_{3} \mathrm{Al}_{2} \mathrm{C}$ and $\mathrm{Ta}_{5} \mathrm{Al}_{3} \mathrm{C}$ were identified. The morphology, phase composition and preferred orientation of the films deposited were found to be highly sensitive to the Ta fraction of the incident flux during deposition. No MAX phase material was observed, indicating that the Ta-containing MAX phases do not form under the deposition conditions investigated. Explanations associated with inadequate coverage of stochiometries, preferential sputtering, and thermodynamic instability have been ruled out. An explanation based on reduced surface diffusion of Ta during growth is proposed. A substantially higher substrate temperature during deposition is likely to be required to synthesize Ta-containing MAX phases.
\end{abstract}

\section{Introduction}

The tantalum $\mathrm{M}_{n+1} \mathrm{AX}_{n}$ (MAX) phases are of fundamental interest as tantalum has the largest atomic mass of any of the transition metals that form MAX phases, and so the Ta MAX phases have some extreme properties among these materials. Experimental investigations of bulk $\mathrm{Ta}_{2} \mathrm{AlC}$ and $\mathrm{Ta}_{4} \mathrm{AlC}_{3}[1,2]$ have found and reported the highest bulk moduli of the MAX phases, and the elastic constants of several of the Ta-containing MAX phase structures are predicted to have extreme values [3].

The MAX phases $\mathrm{Ta}_{2} \mathrm{AlC}, \mathrm{Ta}_{3} \mathrm{AlC}_{2}$ and $\mathrm{Ta}_{4} \mathrm{AlC}_{3}$ have been synthesized by bulk high-temperature methods $[1,2,4,5]$ and by crystal growth in a molten $\mathrm{Al}$ solvent [6]. These phases have not, however, been reported as thin films although thin film synthesis has been attempted [7]. More generally, there have been no reports of the thin film synthesis of any MAX phases with a period 6 transition metal, Ta and Hf being the two possibilities. It has been suggested that the apparent difficulty of growing these phases as thin films could be a consequence of the high atomic mass of Ta and $\mathrm{Hf}$, resulting in increased resputtering of the deposited film by Ar atoms reflected off the transition metal target [7].

We describe experiments conducted with the aim of determining whether the tantalum MAX phases form as thin films using magnetron sputtering, under conditions similar to those used for the deposition of other MAX phases. In particular, we aim to determine whether resputtering is a process that shifts the composition of the deposited material preventing formation of the MAX phase. To efficiently explore a wide range of Ta-Al-C

\footnotetext{
Thin Solid Films 558, 2 May 2014, pp.

http://doi.org/10.1016/j.tsf.2014.02.086

Email address: mark. tucker@liu.se (Mark D. Tucker)
}

99-103,

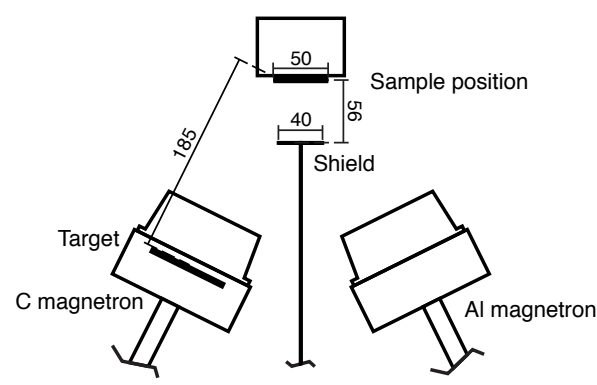

Figure 1: The positions of two of the magnetron sources, the substrate and the deposition shield in the sputtering chamber. The magnetron containing the Ta target is not shown. Dimensions are in $\mathrm{mm}$.

compositions we used a combinatorial method, where a range of compositions are synthesized simultaneously. Combinatorial methods are widely used for investigations of large parameter spaces, and have a long history of use in thin-film research on ternary material systems $[8,9,10]$.

\section{Experimental methods}

Samples were deposited using a sputtering system fitted with four $76 \mathrm{~mm}$ circular magnetrons positioned symmetrically around the substrate in a confocal arrangement. Three elemental targets, of Ta, Al, and C (graphite), were used. The $\mathrm{Al}$ and $\mathrm{C}$ targets were mounted in opposite magnetrons, the Ta target in one of the remaining magnetrons, and the fourth magnetron was not used. The Al and Ta targets were sputtered using DC bias; the $\mathrm{C}$ target was sputtered using RF bias. All three supplies were operated in power regulating mode. 
To produce a composition gradient across the substrate, a square shield was installed in the chamber in front of the substrate, as shown in Figure 1. Trial tantalum depositions were made, varying the size and position of the shield, and surface profilometry was used to assess the resulting film thickness gradients. The shield size and position shown in Figure 1 produced the largest and most linear thickness gradient across the substrate of the geometries tested.

The thickness deposition rate as a function of position across the substrate was determined for each magnetron target by depositing test samples at ambient temperature and making stylus profilometer thickness measurements at a grid of points on the sample surface. Quartz crystal monitor (QCM) measurements were made to determine the film density and deposition rate as a function of magnetron power for each target.

Following these calibration steps, four Ta-Al-C samples were made at magnetron power levels chosen to produce a sample set covering the area of interest in the Ta-Al-C incident composition space. For the Ta and $\mathrm{Al}$ targets, the power values used were in the ranges $60-170 \mathrm{~W}$ and $30-240 \mathrm{~W}$ respectively. For the $\mathrm{C}$ target, $600 \mathrm{~W}$ was used for all samples. The substrates used were polished single crystal (0001) oriented $\mathrm{Al}_{2} \mathrm{O}_{3}$ wafers of $50 \mathrm{~mm}$ diameter. The substrate was clamped to a sample holder held at $850{ }^{\circ} \mathrm{C}$ during deposition. Sputtering was carried out with $\mathrm{Ar}$ at a pressure of 2.5 mtorr $(0.33 \mathrm{~Pa})$. Each deposition was for 60 minutes. The base pressure in the chamber, after heating the sample, was less than $5 \times 10^{-6}$ torr $\left(7 \times 10^{-4}\right.$ $\mathrm{Pa}$ ); the oxygen content of one of the samples was subsequently measured using X-ray photoelectron spectroscopy and found to be below 2 at. $\%$.

$\mathrm{X}$-ray diffraction (XRD) wafer mapping measurements in $\theta-2 \theta$ geometry were made using a Philips X'Pert MRD diffractometer equipped with a sample stage with $\mathrm{x}$ and $\mathrm{y}$ translation. The illuminated spot on the sample, using line focus with an $\mathrm{x}$-ray mirror, was of dimensions $5 \mathrm{~mm}$ by $\sim 1 \mathrm{~mm}$. Scans over the $2 \theta$ range $5^{\circ}-95^{\circ}$ were taken on a $3 \times 3$ point grid across each sample, with adjacent points separated by a distance of 15 $\mathrm{mm}$. Scans over the smaller $2 \theta$ range $34^{\circ}-48^{\circ}$ were then taken on a finer $9 \times 9$ point grid, with adjacent points separated by a distance of $5 \mathrm{~mm}$. This smaller $2 \theta$ range was chosen to include the main peaks seen in the previous scans.

Areas of the deposited films where the Al fraction of the incident flux was low had a specular visual appearance, whereas areas where the $\mathrm{Al}$ incident flux was higher scattered incident light. To investigate these variations, photographs were taken of the samples using a camera positioned with its axis normal to the sample surface. The sample was illuminated with an electronic flash at oblique incidence to the sample surface. With this arrangement, regions of the sample where light is scattered at the surface appear as light areas in the image, and both specularly reflecting and absorbing regions of the sample appear as dark areas.

Scanning electron microscope (SEM) images were acquired using a Zeiss EVO 50 SEM, operated at $15 \mathrm{kV}$. Images were acquired in plan view, and with a $30^{\circ}$ sample tilt in some areas to reveal the surface topography.

\section{Results}

XRD scans extracted from the wafer maps for the $2 \theta$ range $34^{\circ}-48^{\circ}$ are shown in Figure 2. The scans shown were chosen to include all the peaks seen in the complete wafer map dataset. Two binary carbides were found: $\mathrm{TaC}$ (the cubic monocarbide) and $\mathrm{Ta}_{2} \mathrm{C}$ (the hcp subcarbide). Several peaks corresponding to the intermetallic tetragonal phase $\mathrm{TaAl}_{3}$ are present. The (non-MAX phase) ternary compounds $\mathrm{Ta}_{5} \mathrm{Al}_{3} \mathrm{C}$ and $\mathrm{Ta}_{3} \mathrm{Al}_{2} \mathrm{C}$ are present in small regions. Finally, bcc $\alpha$-Ta was found, the usual phase of bulk Ta. A low intensity background peak is present in the scan at incident composition $\mathrm{Ta}_{21} \mathrm{Al}_{62} \mathrm{C}_{17}$ as this scan was at a point near the edge of the wafer.

The scans over the $2 \theta$ range $5^{\circ}-95^{\circ}$ (not shown) did not indicate the presence of any additional phases. In particular, the distinctive $(00 l)$ MAX phase peaks at low $2 \theta$ angles did not appear in any of the scans.

To further investigate patterns in the composition-space distribution of the phases identified, a list of peak heights and positions was compiled from the XRD data. A principal set of peaks were selected for analysis: $\alpha$-Ta (110), TaC (111), Ta $2 \mathrm{C}(002)$, $\mathrm{TaAl}_{3}$ (112) and (200), $\mathrm{Ta}_{3} \mathrm{Al}_{2} \mathrm{C}$ (111), and $\mathrm{Ta}_{5} \mathrm{Al}_{3} \mathrm{C}$ (002). The $\mathrm{XRD}$ peak intensity as a function of wafer position was combined with the incident composition versus wafer position to generate Figure 3, where XRD peak intensities are plotted as a function of incident composition. In this figure, the region of the incident composition space where a particular phase is found is indicated by discs of a particular colour associated with that phase. The area of each disc is proportional to the intensity of the corresponding XRD peak. Each phase identified in Figure 3 occurs where the incident composition is close to the stoichiometry of that phase.

Figure 4 shows a scattered-light photograph overlaid with XRD peak height data (shown as discs with areas proportional to the XRD peak intensities, as in Figure 3) and contours indicating the incident composition profile across the wafer. Strongly scattering regions are present, some with sharp, well-defined boundaries. The main scattering region in all the samples deposited was defined by an incident Ta concentration of $<35 \%$ and an $\mathrm{Al}: \mathrm{C}$ ratio of $>0.75$; the film outside this region was of specular appearance.

Several of the scattering feature boundaries present in the light scattering photographs run parallel to the Ta concentration contour lines, and these boundaries correspond to sudden changes in the intensity of certain diffraction peaks. In Figure 4 this is most obvious on crossing into the region of strong scattering bounded by the $35 \%$ Ta contour line, where the $\mathrm{TaC}$ and $\mathrm{TaAl}_{3}$ peaks suddenly increase in intensity.

The SEM showed that the variations observed in light scattering across the samples were caused by dramatic differences in the surface structure of the film across the wafer. The film regions of specular appearance appeared smooth and featureless. The region of the wafer between the the $20 \%$ and $35 \%$ Ta contour lines showed a surface structure of columns approximately $200 \mathrm{~nm}$ in diameter: a representative image of these structures is shown in Figure 4 inset A. The columns are tilted from perpendicular to the substrate plane, towards the position of the $\mathrm{Ta}$ 


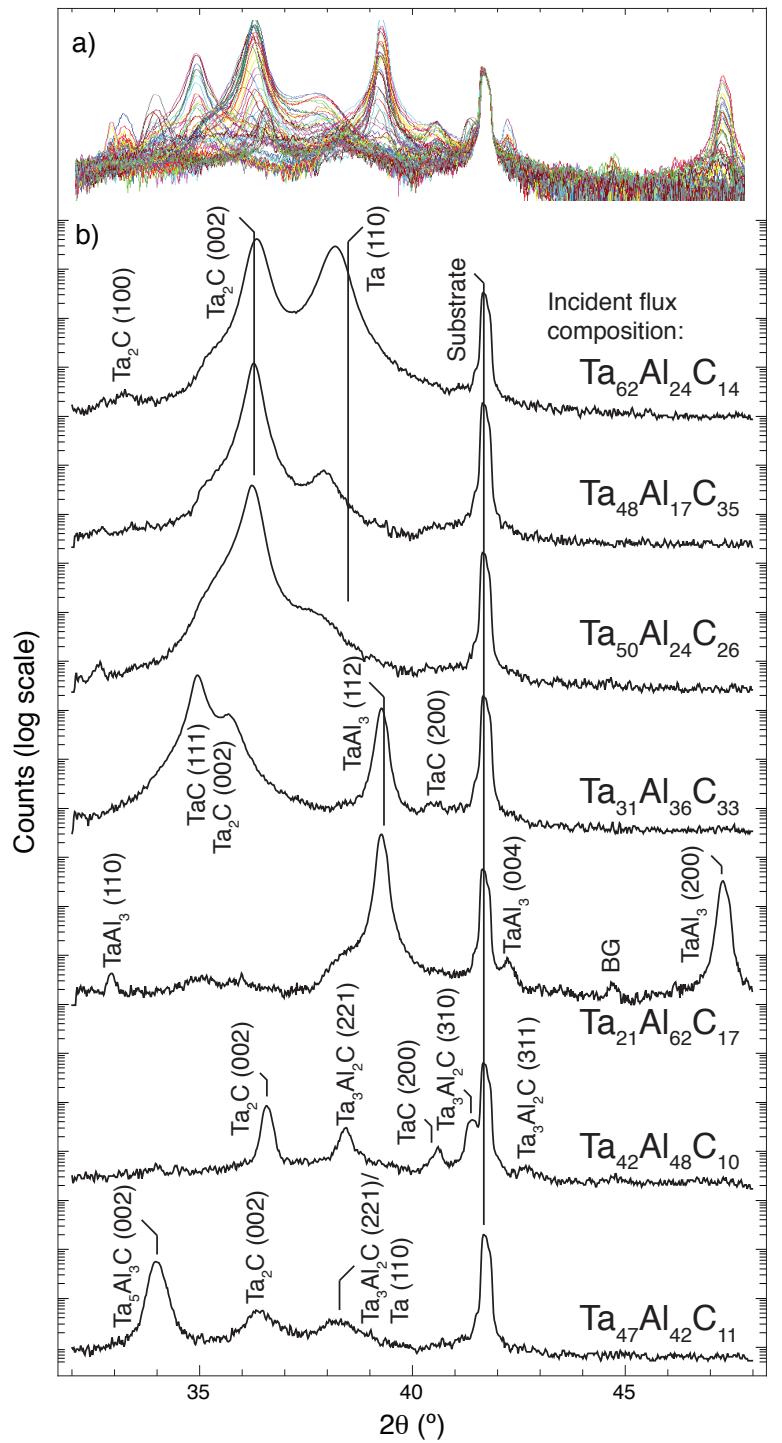

Figure 2: a) The data of one of the 81-scan XRD wafer maps. b) $\theta-2 \theta$ XRD scans taken at points of the indicated incident compositions, extracted from the 81-scan wafer maps. Note that the incident composition is not necessarily the film composition. The scans shown were chosen to include all the peaks seen in the complete dataset. ' $\mathrm{BG}$ ' in the $\mathrm{Ta}_{21} \mathrm{Al}_{62} \mathrm{C}_{17}$ scan is a background peak from the sample stage.

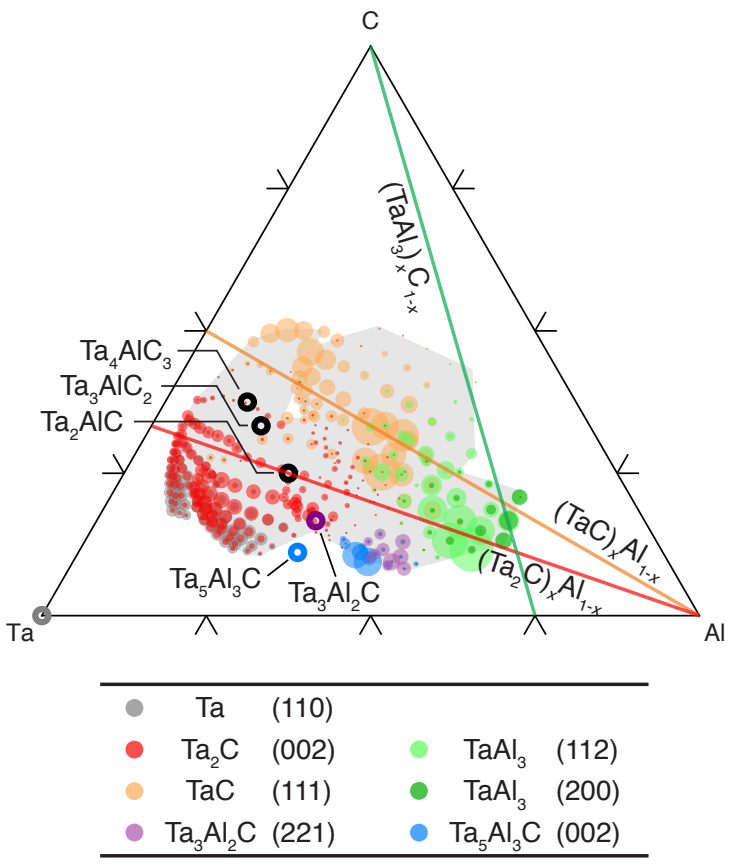

Figure 3: (Colour online) The measured intensity of XRD peaks, corresponding to several phases present in the films, plotted in the ternary composition space determined from the incident flux. The area of each disc plotted is proportional to the height of a diffraction peak measured at that point, with the scaling for each phase chosen for clarity. The open circles and coloured lines indicate the positions where the incident composition corresponds to the stoichiometry of the phases detected. The light grey region is the area of the composition space investigated. 

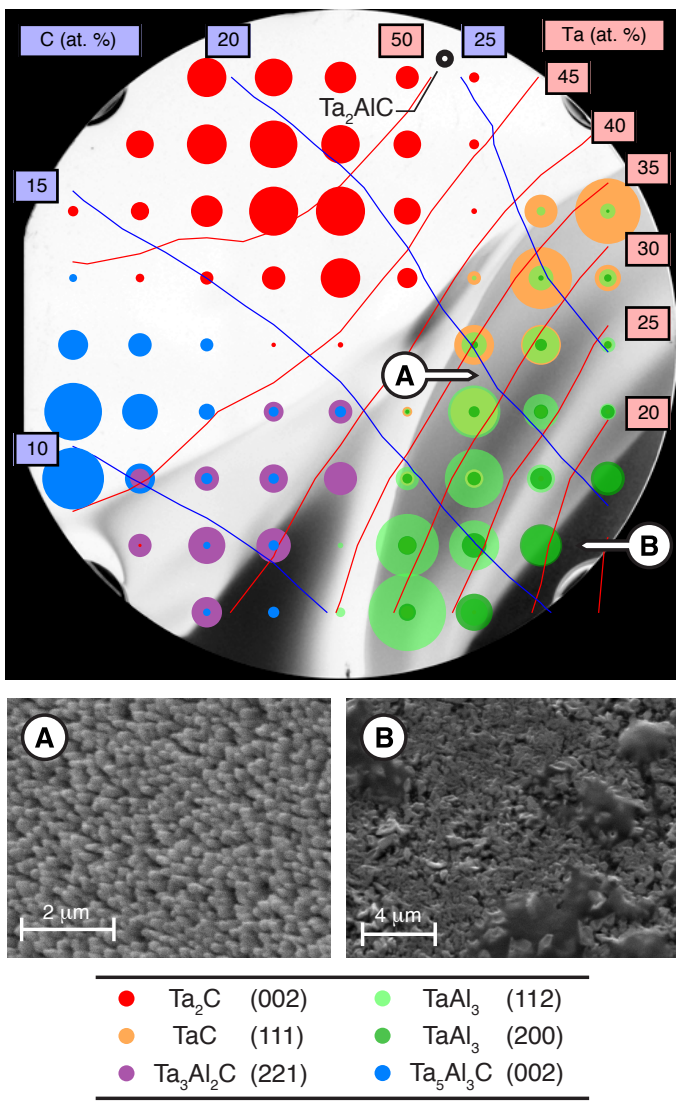

Figure 4: (Colour online) The light scattering photograph of the sample of highest $\mathrm{Al}$ content, deposited with magnetron power setpoints of $150 \mathrm{~W}$ (Ta), 240 $\mathrm{W}(\mathrm{Al})$ and $600 \mathrm{~W}(\mathrm{C})$. The photograph is overlaid with XRD peak intensity data and incident composition contours to show the relationships between incident composition, phase makeup and morphology of the film. The area of each disc plotted is proportional to the height of a diffraction peak measured at that point, with the scaling for each phase chosen for clarity. The greyscale of the photograph of the wafer has been inverted so that scattering regions appear dark. The open circle indicates the wafer position where the incident composition was $\mathrm{Ta}_{2} \mathrm{AlC}$. Insets $\mathrm{A}$ and $\mathrm{B}$ are SEM micrographs taken at the positions indicated in the main figure. source during growth of the film. As the Ta concentration decreases across the wafer to below approximately $20 \%$, a different surface morphology of crystallites and rounded droplet-like features suddenly appears. An SEM image from this region is shown in Figure 4 inset B. Comparison of EDX measurements (not shown) and the XRD data in this region indicates that the crystallites are $\mathrm{TaAl}_{3}$ and the droplets are elemental $\mathrm{Al}$.

\section{Discussion}

\subsection{Phases and preferred orientation}

The bcc $\alpha$ phase of Ta is observed in these samples, as expected given that the substrate temperature during deposition was well above the $\beta$ to $\alpha$ transition temperature[11]. The (110) preferred orientation of the Ta phase has been observed in films grown on polycrystalline steel substrates [12] and so is a fibre texture rather than an epitaxial relationship between the film and the substrate.

Several other phases visble in the XRD data show strong texture. The strong (002) $\mathrm{Ta}_{2} \mathrm{C}$ and $\mathrm{Ta}_{5} \mathrm{Al}_{3} \mathrm{C}$ peaks suggest basal plane texture of these phases. The $\mathrm{TaC}$ (111) peak suggests epitaxy with the (0001) $\mathrm{Al}_{2} \mathrm{O}_{3}$ substrate, as (111) orientation through epitaxy is commonly observed for transition metal monocarbide films deposited on hexagonal substrate surfaces. The $\mathrm{TaAl}_{3}$ (112) peak at $2 \theta=39.3^{\circ}$ suggests (112) preferred orientation caused by alignment of the approximately hexagonal (strictly, rhombic) lattice of the $\mathrm{TaAl}_{3}$ (112) surface with the hexagonal lattice of the substrate.

The $\mathrm{Ta}_{2} \mathrm{C}(001)$ and $\mathrm{Ta}(110)$ phases are shifted to larger $\mathrm{d}$-spacings in some of the XRD scans. We speculate that these shifts are due to the presence of a solid solution of $\mathrm{Al}$ in the $\mathrm{Ta}_{2} \mathrm{C}$ and $\mathrm{Ta}$ phases. This is consistent with the gradual shift of these peaks observed in scans (not shown) at intermediate compositions.

\subsection{Surface morphology}

Along the 0.35 Ta fraction contour shown in Figure 4, (111) oriented $\mathrm{TaC}$ is present in greater quantity at lower $\mathrm{Al}: \mathrm{C}$ ratios, and randomly oriented $\mathrm{TaAl}_{3}$ is strongest at high $\mathrm{Al}: \mathrm{C}$ ratios. The surface features shown in Figure 4 inset (A) were observed in both regions. Their formation is triggered suddenly as the incident Ta flux fraction is reduced past 0.35.

These features are likely to be the tops of columnar grains, consistent with the predictions of structure zone models often used to classify thin film growth. A critical parameter in these models is the ratio $T_{s} / T_{m}$, where $T_{s}$ is the substrate temperature during deposition and $T_{m}$ is the melting point of the film material [13]. Higher values of $T_{s} / T_{m}$ are associated with the occurrence of recrystallization and the growth of large grains, whereas low values are associated with the growth of separated columnar structures, similar to those observed here, as a consequence of low adatom mobility. TaC, which is present in the columnar growth regions, has an extremely high melting point, of about $3880{ }^{\circ} \mathrm{C}$. Because of this, the ratio $T_{s} / T_{m}$ is fairly low for $\mathrm{TaC}$ in spite of the rather high substrate temperature (850 ${ }^{\circ} \mathrm{C}$ ) used during deposition. The observed tilt of the columns 
towards the Ta magnetron source (that is, in the direction of the Ta flux) is consistent with shadowing of the deposition flux affecting the structure, as expected in the case of low $T_{s} / T_{m}$.

Regions of $\mathrm{TaC}$ growth in the samples grown with lower incident $\mathrm{Al}$ fraction (not shown in Figure 4) do not display the columnar morphology and instead show a surface that is smooth to the resolution limit of the SEM.

\subsection{On the absence of MAX phase growth}

The (111) oriented MX monocarbide has generally been found to trigger epitaxial (0001) growth of the corresponding $\mathrm{M}_{n+1} \mathrm{AX}_{n}$ phase [14], a result of the close correlation between the M-M distance of the carbide and the a-lattice parameter of the MAX phase [15]. Although (111) TaC was observed, MAX phase peaks were not present in any of the XRD scans.

A trivial explanation for the absence of MAX phase in the samples investigated here would be that an inadequate part of the incident composition space was investigated. The phases present in the films demonstrate that the samples do, in fact, cover a sufficiently large part of the composition space. The phases observed have elemental ratios bounding those of the $\mathrm{Ta}_{n+1} \mathrm{AlC}_{n}$ MAX phases, with Ta:C ratios from 1:0 (Ta) to 1:1 $(\mathrm{TaC})$ and Ta:Al ratios from 1:0 (Ta) to $1: 3\left(\mathrm{TaAl}_{3}\right)$. The three $\mathrm{Ta}_{n+1} \mathrm{AlC}_{n}$ MAX phases have Ta:C ratios in the range from 1:0.5 to $1: 0.75$, and Ta:Al ratios from 1:0.25 to 1:0.5. As the film composition space can be verified in this manner, it is unnecessary to separately analyse and map the film composition of the samples to conclude that films covering the correct region of the composition space were deposited.

One factor suggested as a possible explanation of the lack of success in attempts at $\mathrm{Ta}_{n+1} \mathrm{AlC}_{n}$ thin film growth is resputtering of the deposited film by energetic Ar atoms [7]. Ar atoms reflected off the sputtering target can have high initial energies, of several hundred $\mathrm{eV}$, depending on the target bias, and sputtering of the growing film by these atoms could upset the stoichiometry of the film. However at the process pressure used, the path length from target to substrate is large compared to the mean free path of an energetic Ar atom, and on average a reflected Ar atom will collide several times with background gas Ar atoms before it reaches the substrate [16]. These collisions between equal masses maximize kinetic energy transfer and so efficiently reduce the energy of the reflected Ar atoms. If selective resputtering of an element was occurring, phases would be found displaced from their expected location in the incident composition diagram (Figure 3): if, for example, Al was being resputtered, the Al-containing phases would be displaced towards the $\mathrm{Al}$ vertex. The fact that the observed phases are present where expected in the figure demonstrates that selective resputtering does not occur to an extent that substantially alters the composition of the deposited material from the composition of the incident flux.

Bulk synthesis of the $\mathrm{Ta}_{n+1} \mathrm{AlC}_{n}$ MAX phases, and indeed of most MAX phases, requires temperatures of $1500-1600{ }^{\circ} \mathrm{C}$ to be maintained for many hours. Thin film MAX phase growth is usually possible at much lower temperatures than are required for synthesis of the same phase in bulk, usually in the range $600-1000{ }^{\circ} \mathrm{C}$. It appears that this is not the case for the
$\mathrm{Ta}_{n+1} \mathrm{AlC}_{n}$ MAX phases: a higher substrate temperature may be required for their growth as thin films.

One possibility is that the $\mathrm{Ta}_{n+1} \mathrm{AlC}_{n}$ MAX phases are thermodynamically unstable in comparison to a mixture of competing phases at the growth temperature we used, $850{ }^{\circ} \mathrm{C}$. However, $\mathrm{Ta}_{2} \mathrm{AlC}$ is included in a published ternary phase diagram (isothermal section at $700{ }^{\circ} \mathrm{C}$ [17]), suggesting that the $\mathrm{Ta}_{2} \mathrm{AlC}$ phase (at least) should be stable at $850^{\circ} \mathrm{C}$. For comparison, the archetypal MAX phases $\mathrm{Ti}_{3} \mathrm{SiC}_{2}$ and $\mathrm{Cr}_{2} \mathrm{AlC}$ appear in ternary phase diagram isothermal sections at $1100{ }^{\circ} \mathrm{C}\left(\mathrm{Ti}_{3} \mathrm{SiC}_{2}\right)$ [18] and $800{ }^{\circ} \mathrm{C}\left(\mathrm{Cr}_{2} \mathrm{AlC}\right)$ [19] and can be grown by sputter deposition at substrate temperatures in this range.

Alternatively, a kinetic limitation on the growth of the $\mathrm{Ta}_{n+1} \mathrm{AlC}_{n}$ MAX phases is suggested by the expected reduced surface and bulk diffusion of the heavy Ta atoms, compared to lighter Melements. The surface diffusion rate may the more significant, given the step-flow mechanism of MAX phase film growth[14]. Because the elemental constituents of a MAX phase must segregate into layers, the average distance an adsorbed atom must travel before finding its final site in a MAX phase structure will be greater than for simpler phases. The surface mobility of an adatom is determined by the jump 'attempt rate' for hops from one binding site on the surface to another and the probability of success. For a simple analysis, assuming a fixed kinetic energy per adatom, we can consider an attempt rate proportional to $\sqrt{1 / m}$. Then the expected surface diffusion rate of Ta would be about half of that of the typical lighter M-element Ti, given the atomic masses of Ta (180 amu) and Ti (48 amu). The same is true for the other possible MAX phase constituents in period 6- $\mathrm{Hf}, \mathrm{Tl}$ and $\mathrm{Pb}$ - whose MAX phases have also only been synthesized in bulk form.

\section{Conclusion}

A combinatorial study was carried out to explore synthesis of thin films in the Ta-Al-C composition space, in order to identify whether the $\mathrm{Ta}_{n+1} \mathrm{AlC}_{n}$ MAX phase materials can be synthesized as thin films by magnetron sputtering. The phases $\alpha$-Ta, Al, TaC, Ta 2 C, TaAl, $\mathrm{Ta}_{5} \mathrm{Al}_{3} \mathrm{C}$, and $\mathrm{Ta}_{3} \mathrm{Al}_{2} \mathrm{C}$ were identified. The phases present were found to be highly sensitive to the tantalum fraction of the flux incident during deposition. None of the $\mathrm{Ta}_{n+1} \mathrm{AlC}_{n}$ MAX phases were found, even though the presence of an epitaxial MX carbide, as found, is often a trigger for MAX phase growth, and the composition-space locations of the other phases found demonstrate that an adequate area of the composition space was investigated.

These results suggest that the Ta-containing MAX phases cannot be synthesized using thin-film deposition conditions similar to those routinely used to deposit other MAX phases. Previous attempts at synthesis of the Ta-containing phases using cosputtering have also failed to produce these MAX phases. As a result of our work, the suggestion that resputtering effects are responsible for the absence of these phases in sputtered films is not supported since the correct stoichiometry has been achieved. The absence of MAX phase growth may be explained by reduced surface diffusion of the M-element during growth, and a substantially higher substrate temperature during 
deposition is likely to be required: unfortunately such a high temperature regime is difficult to achieve in sputter deposition with commercially available heater designs.

\section{Acknowledgments}

Funding for this work was provided by the Australian Research Council.

[1] B. Manoun, S. K. Saxena, T. El-Raghy, M. W. Barsoum, High-pressure xray diffraction study of $\mathrm{Ta}_{4} \mathrm{AlC}_{3}$, Applied Physics Letters 88 (20) (2006) 201902.

[2] B. Manoun, R. P. Gulve, S. K. Saxena, S. Gupta, M. W. Barsoum, C. S. Zha, Compression behavior of $\mathrm{M}_{2} \mathrm{AlC}(\mathrm{M}=\mathrm{Ti}, \mathrm{V}, \mathrm{Cr}, \mathrm{Nb}$, and $\mathrm{Ta}$ ) phases to above $50 \mathrm{GPa}$, Physical Review B 73 (2).

[3] M. F. Cover, O. Warschkow, M. M. M. Bilek, D. R. McKenzie, A comprehensive survey of $\mathrm{M}_{2} \mathrm{AX}$ phase elastic properties, Journal of Physics: Condensed Matter 21 (30) (2009) 305403.

[4] W. Jeitschko, H. Nowotny, F. Benesovsky, Kohlenstoffhaltige ternäre Verbindungen (H-Phase), Monatshefte für Chemie / Chemical Monthly 94 (4) (1963) 672-676.

[5] P. Eklund, J. P. Palmquist, J. Howing, D. H. Trinh, T. El-Raghy, H. Hogberg, L. Hultman, $\mathrm{Ta}_{4} \mathrm{AlC}_{3}$ : Phase determination, polymorphism and deformation, Acta Materialia 55 (14) (2007) 4723-4729.

[6] J. Etzkorn, M. Ade, H. Hillebrecht, $\mathrm{Ta}_{3} \mathrm{AlC}_{2}$ and $\mathrm{Ta}_{4} \mathrm{AlC}_{3}$ - singlecrystal investigations of two new ternary carbides of tantalum synthesized by the molten metal technique, Inorganic Chemistry 46 (4) (2007) 14101418.

[7] P. Eklund, M. Beckers, U. Jansson, H. Hogberg, L. Hultman, The $\mathrm{M}_{n+1} \mathrm{AX}_{n}$ phases: Materials science and thin-film processing, Thin Solid Films 518 (8) (2010) 1851-1878.

[8] K. Kennedy, T. Stefansky, G. Davy, V. F. Zackay, E. R. Parker, Rapid method for determining ternary-alloy phase diagrams, Journal of Applied Physics 36 (12) (1965) 3808-3810.

[9] R. B. van Dover, L. D. Schneemeyer, R. M. Fleming, Discovery of a useful thin-film dielectric using a composition-spread approach, Nature 392 (6672) (1998) 162-164.

[10] R. Mertens, Z. M. Sun, D. Music, J. M. Schneider, Effect of the composition on the structure of $\mathrm{Cr}$-Al-C investigated by combinatorial thin film synthesis and ab initio calculations, Advanced Engineering Materials 6 (11) (2004) 903-907.

[11] M. H. Read, C. Altman, A new structure in tantalum thin films, Applied Physics Letters 7 (3) (1965) 51-52.

[12] S. L. Lee, M. Doxbeck, J. Mueller, M. Cipollo, P. Cote, Texture, structure and phase transformation in sputter beta tantalum coating, Surface and Coatings Technology 177-178 (2004) 44-51.

[13] J. A. Thornton, The microstructure of sputter-deposited coatings, Journal of Vacuum Science \& Technology A: Vacuum, Surfaces, and Films 4 (6) (1986) 3059-3065.

[14] J. Emmerlich, H. Hogberg, S. Sasvari, P. O. A. Persson, L. Hultman, J. P. Palmquist, U. Jansson, J. M. Molina-Aldareguia, Z. Czigany, Growth of $\mathrm{Ti}_{3} \mathrm{SiC}_{2}$ thin films by elemental target magnetron sputtering, Journal of Applied Physics 96 (9) (2004) 4817-4826.

[15] M. W. Barsoum, The $\mathrm{M}_{n+1} \mathrm{AX}_{n}$ phases: A new class of solids; thermodynamically stable nanolaminates, Progress in Solid State Chemistry 28 (14) (2000) 201-281.

[16] W. D. Westwood, Calculation of deposition rates in diode sputtering systems, Journal of Vacuum Science and Technology 15 (1) (1978) 1-9.

[17] J. C. Schuster, Aluminium-Carbon-Tantalum, in: Ternary Alloys, Vol. 3, VCH, 1990, pp. 549-553.

[18] W. J. J. Wakelkamp, F. J. J. van Loo, R. Metselaar, Phase relations in the Ti-Si-C system, Journal of the European Ceramic Society 8 (3) (1991) 135-139.

[19] J. C. Schuster, H. Nowotny, C. Vaccaro, The ternary systems: Cr-Al-C, V-Al-C, and Ti-Al-C and the behavior of H-phases $\left(\mathrm{M}_{2} \mathrm{AlC}\right)$, Journal of Solid State Chemistry 32 (2) (1980) 213-219. 